\title{
Parallel fluorescence and phosphorescence monitoring of singlet oxygen photosensitization in rats
}

\author{
Roman Dědic*, Adam Stíbal and Vojtěch Vyklický \\ Faculty of Mathematics and Physics \\ Department of Chemical Physics and Optics \\ Charles University in Prague \\ Ke Karlovu 3, 12116 Praha 2, Czech Republic \\ *Roman.Dedic@mff.cuni.cz \\ Miloslav Franěk \\ Third Faculty of Medicine \\ Department of Normal, Pathological and Clinical Physiology \\ Charles University in Prague \\ Ke Karlovu 4, 12000 Praha 2, Czech Republic \\ Antonín Svoboda and Jan Hála \\ Faculty of Mathematics and Physics \\ Department of Chemical Physics and Optics \\ Charles University in Prague \\ Ke Karlovu 3, 12116 Praha 2, Czech Republic
}

Received 26 February 2015

Accepted 13 April 2015

Published 21 May 2015

\begin{abstract}
The time- and spectral-resolved set-up for measurements of weak infrared luminescence of photosensitizers (PSs) and singlet oxygen using optical lightguides was used on skin of laboratory animals in vivo. Wistar rats with abdominal incisions treated by methylis aminolevulinitis (MAL) were used as a model. A control group of animals with abdominal incisions was also tested. Spectrally resolved fluorescence of the PS was acquired during the treatment from the same spot. The intensity and spectral profile of the fluorescence signal from the skin can be used to guide the detection setup to the investigated spots in the lesion. The rate of bleaching of Protoporphyrin IX band and appearance of a band of its photoproducts during the treatment can be characterized by the exposition $E_{D}$, under which the latter becomes dominant feature in fluorescence spectrum. $E_{D}$ value differs statistically significantly between the normal skin and the lesion treated by MAL. No direct proportionality was found between the fluorescence signal and singlet oxygen production. Nevertheless, the strong fluorescence signal is necessary but not a sufficient condition for higher singlet oxygen production in vivo. $E_{D}$ value correlates rather well with production of
\end{abstract}

${ }^{*}$ Corresponding author.

This is an Open Access article published by World Scientific Publishing Company. It is distributed under the terms of the Creative Commons Attribution 3.0 (CC-BY) License. Further distribution of this work is permitted, provided the original work is properly cited. 
singlet oxygen, but differently in lesion and normal skin. Lifetimes of singlet oxygen differ between spots outside and in the lesion. PS triplet state lifetimes exhibit weak difference between spots treated and untreated by MAL.

Keywords: PDT dosimetry; singlet oxygen; Protoporphyrin IX; time-resolved luminescence; photodiagnosis.

\section{Introduction}

Photodynamic therapy (PDT) represents a powerful modality for treatment of various chronic diseases including oncologic ones. PDT is based on selective uptake of a drug called photosensitizer (PS) in the lesion due to different morphological or physiological properties of the diseased tissue. PS, which is nontoxic in dark, produces toxic species under local irradiation of the lesion by light. The light brings PS into the singlet excited state, which may undergo inter-system crossing to the triplet state. The long-lived triplet state, whose relaxation back to the ground state is spin-forbidden, may give rise to various kinds of free radicals which cause oxidative damage to the treated tissue in so called photoeffect of the first type. Alternatively, the triplet excited state of PS transfers its energy in a spin-allowed process to molecular oxygen, whose ground state is also triplet, giving rise to the excited singlet state of oxygen, generally called only singlet oxygen $\left({ }^{1} \mathrm{O}_{2}\right) \cdot{ }^{1} \mathrm{O}_{2}$ exhibits several orders higher reactivity toward various biological molecules (such as lipids, proteins or nucleic acids) than ground state oxygen. It causes severe oxidative damage to the cellular environment. ${ }^{1} \mathrm{O}_{2}$ is believed to be the main cytotoxic agent in PDT. ${ }^{1}$ The oxidative damage usually starts as a cascade of processes ending in cellular death of the cells in the treated lesion. The damage caused by production of ${ }^{1} \mathrm{O}_{2}$ is called photoeffect of the second type. The double selectivity of PDT due to the selective uptake of PS and the localized irradiation of the lesion together with dark nontoxicity and relatively fast clearance rate of the used PS are reasons for very low side effects of the treatment. Together with very good cosmetic outcome of the treatment, these properties make PDT a perfect treatment modality of various oncologic diseases, mainly for diagnoses, where high-dose radiotherapy or mutilating surgery treatment are otherwise required.

A strong light-dose dependence of the therapeutic effect is a characteristic for PDT with so called threshold effect between undamaged and apoptotic/necrotic tissue. Existence of a minimal ${ }^{1} \mathrm{O}_{2}$ concentration needed to provoke the biologic effects in the cells is highly probable. The threshold may be utilized to further minimize the effect of the treatment on adjacent tissues while maintain the high therapeutic response of the lesion. ${ }^{2}$ To some extent, the total PDT dose may be utilized to select primary biological targets of the photoeffect and modulate the therapeutic mechanism. ${ }^{3}$ Unfortunately, the tissue responses to the same PDT dose are highly heterogeneous between individual patients, lesions, and even within single target volume due to differences in local PS concentration, light penetration, or PDT-induced hypoxia. The variability of response makes on-line monitoring of the tissue response to the treatment, so called PDT dosimetry, necessary for further exploration the possible benefits of the therapy by individual modulation of the treatment to individual lesions.

Three approaches to the PDT dosimetry are possible: The traditional explicit dosimetry combines the knowledge of amounts of light, PS, and ground state oxygen in a model allowing to evaluate the amount of generated ${ }^{1} \mathrm{O}_{2}$. The limitations of this method were discussed earlier. ${ }^{4,5}$ Currently, a novel technique utilizing measurements of either time-resolved or steady-state fluorescence of the PS to measure local oxygen concentration was reported. ${ }^{6}$ In contrast, the implicit dosimetry employs monitoring of photoproduct formation or photobleaching of PS in reaction with ${ }^{1} \mathrm{O}_{2}$ to measure the quantity of ${ }^{1} \mathrm{O}_{2}$ produced. However, certain ${ }^{1} \mathrm{O}_{2}$-independent reactions may contribute to the same effect, such as PS photobleaching in reaction of PS triplet with biological targets. ${ }^{7-9}$ Recently, a correlation between PpIX fluorescence and ${ }^{1} \mathrm{O}_{2}$ luminescence signal in 5-aminolevulinic acid (ALA) PDT treated healthy human skin was published ${ }^{10}$ and fluorescence-based metrics were used to predict clinical phototoxic response (erythema). However, a failure of the implicit dosimetry under hypoxic conditions 
was reported in a study, where simultaneous measurements of PS bleaching and direct detection of ${ }^{1} \mathrm{O}_{2}$ (will be discussed later) were evaluated and correlated with cytotoxicity on suspensions of AML-5 leukemia cells. ${ }^{11}$

The third possibility is utilization of optical detection of ${ }^{1} \mathrm{O}_{2}$. Besides the indirect methods of EPR, absorption or fluorescence spectroscopy employing specific ${ }^{1} \mathrm{O}_{2}$ probes, direct detection of weak infrared phosphorescence of ${ }^{1} \mathrm{O}_{2}$ around $1274 \mathrm{~nm}$ may be used for detection and even imaging of ${ }^{1} \mathrm{O}_{2}$ production. ${ }^{12,13}$ The direct detection of the extremely weak phosphorescence of ${ }^{1} \mathrm{O}_{2}$ from in vitro and in vivo biological material was demonstrated, ${ }^{1}$ the cumulative signal of ${ }^{1} \mathrm{O}_{2}$ luminescence was correlated with cell survival in tumor cell suspensions ${ }^{14}$ and even validated on $\delta$-aminolevulinic acid ALAsensitized normal mouse skin. ${ }^{15}$ Recently, the ${ }^{1} \mathrm{O}_{2}$ luminescence signal was correlated with in vivo tumor response in gliosarcoma tumors in dorsal window on mice by Wilson et al. ${ }^{16}$ Finally, imaging of ${ }^{1} \mathrm{O}_{2}$ emission on animals in vivo was reported..$^{17}$ Details of these pioneering attempts are discussed in a review. ${ }^{18}$

The measurements of overall ${ }^{1} \mathrm{O}_{2}$ luminescence serve as a probe of the amount of ${ }^{1} \mathrm{O}_{2}$ produced. Moreover, time-resolved measurements of ${ }^{1} \mathrm{O}_{2}$ luminescence can provide lifetimes of ${ }^{1} \mathrm{O}_{2}$ itself as well as those of PS excited states. The lifetimes reflect interaction of ${ }^{1} \mathrm{O}_{2}$ and PS with the environment. Time- and spectral-resolved detection of infrared emission of ${ }^{1} \mathrm{O}_{2}$ and PS was fruitfully used in our group to investigate their interactions with different proteins, ${ }^{19,20}$ lipids, ${ }^{21,22}$ and liposomes. ${ }^{23,24}{ }^{1} \mathrm{O}_{2}$ luminescence kinetics from suspensions of living cells were reported by several groups. ${ }^{25-29}$ Even detection of ${ }^{1} \mathrm{O}_{2}$ luminescence kinetics from single cells were presented by Ogilby's group. ${ }^{30,31}$ The possibility of using optical lightguides to obtain not only the cumulative signal of ${ }^{1} \mathrm{O}_{2}$ luminescence but also its kinetics with sufficient time-resolution reflecting interactions of PS and ${ }^{1} \mathrm{O}_{2}$ with cellular environment during PDT in layers of living cells in vitro was recently reported by our group. ${ }^{32}$ Time-resolved ${ }^{1} \mathrm{O}_{2}$ kinetics measurements from ex vivo skin of pig ears was recently reported by Schlothauer et al. ${ }^{13}$

In addition to the implicit PDT dosimetry, the fluorescence of PS can be used in fluorescence diagnostics, also known as photodiagnostics, which utilizes elevated concentration of PS in the target tissues for noninvasive analysis of various cutaneous lesions. ${ }^{33}$ This method represents prompt modality for efficient diagnostics of early stages of malignancies, which generally offer, a better prognosis for the patients. Moreover, the visible fluorescence of lesions loaded by PS may be used for demarcation of the lesions in fluorescence-guided tumor resection ${ }^{34}$ or biopsies. ${ }^{35}$

The aim of our contribution is to combine our time- and spectral-resolved set-up for infrared luminescence of PS and ${ }^{1} \mathrm{O}_{2}$ using optical lightguides for collection of the signals from biological samples ${ }^{32}$ with fiber-optics fluorescence spectrometer to a single device. It will enable simultaneous detection of photobleaching of PS and production of its photoproducts with measurements of kinetics of ${ }^{1} \mathrm{O}_{2}$ and PS from the very same spot during photodynamic action. Our approach is similar to the methodology recently published by Schlothauer et $a l .{ }^{13}$ and Mallidi et al. ${ }^{10}$ We report successful usage of the device on actinically altered skin in incisions of laboratory rats in vivo treated by methylester (MAL) of ALA. ALA is a precursor of potent endogenous PS Protoporphyrin IX (PpIX) in biosynthesis of heme. Exogenous application of ALA circumvents feedback regulation of its synthesis in heme biosynthesis pathway and saturates ferrochelatase (an enzyme converting PpIX to heme). Due to lower activity of ferrochelatase accompanied by low availability of iron in fast proliferating cells of cancerous tissues, the biosynthesis of heme is interrupted in the PpIX step. Therefore, PpIX is accumulated in high concentrations in mitochondria of the cells. ${ }^{36}$ ALA and its methylester are widely used in clinical practice for both PDT and photodiagnostics. ${ }^{37,38}$ MAL exhibits higher neoplastic tissue specificity and better tissue penetration in comparison to ALA with equivalent efficacy of the treatment. ${ }^{39,40}$ The neoplastic tissues of healing skin incisions exhibit similar behavior as the tumor cells after application of ALA/MAL and thus were used as a model in this study. ${ }^{33}$

\section{Experimental Methods}

\subsection{Materials}

Abdominal skin incisions were performed in 10 adult male Wistar rats (Velaz, Czech Republic) under ketamine $(100 \mathrm{mg} / \mathrm{kg}$ i.p.) and xylazine $(16 \mathrm{mg} / \mathrm{kg}$ i.p.) anesthesia. Animals were kept at $(22 \pm 2)^{\circ} \mathrm{C}$ (relative humidity was $40-70 \%$ ) under 
12-h light/12-h dark cycle. The lesions were treated by $16 \%$ methylis aminolevulinitis (MAL) (Metvix cream, containing $160 \mathrm{mg} / \mathrm{g}$ of methyl aminolevulinate as hydrochloride) in the experimental group ( 8 animals) 2-3 days after the surgical procedure, whereas the lesions remained untreated in control group (2 animals). Approximately $1 \mathrm{~mm}$ thick layer of Metvix cream was applied to the lesion and its small surroundings. The lesions were covered by bandages to avoid mechanical damage and light exposure before measurements. The measurements were carried out after 3 to $6 \mathrm{~h}$ after the Metvix administration. The animals were kept under ketamine/xylazine anesthesia on a heated mat to maintain their body temperature during the measurements. All experiments were approved by the Committee for Animal Care and Use of the 3rd Faculty of Medicine, Charles University in Prague.

\subsection{Methods}

A special home-made holder which ensures aiming of the individual lightguides to the same spot on the skin of the specimen was developed. The holder consists of one half of a metal cylinder with precisely drilled holes which serve as ports for the lightguides. The lightguides are ended by long metal ferrules which enable tight fit to the individual ports as well as keeping the ends of the lightguides in approximately only $2 \mathrm{~mm}$ distance from the specimen surface (i.e., the skin of the animal). The complete holder equipped by three lightguides raised above the skin of the animal is shown in Fig. 1.

In this case, the three lightguides were used: excitation, fluorescence and near-infrared (NIR) phosphorescence (either PS or ${ }^{1} \mathrm{O}_{2}$ ) detection. The bottom part of the holder is covered by thin metal plate with a small elliptic hole. This plate is in direct contact with the skin of the animal during measurements ensuring constant distance of the lightguides from the skin. It also prevents preirradiation of the lesion area which is not measured by the scattered excitation light.

The $6 \mathrm{~ns}$ long excitation pulses of $\approx 10 \mu \mathrm{J}$ at $420 \mathrm{~nm}$ with $200 \mathrm{~Hz}$ repetition provided by excimer laser-pumped dye laser are emitted from the $0.6 \mathrm{~mm}$ diameter UV/Vis optical fiber (Ocean Optics) perpendicular to the animal skin. This creates a circular spot of diameter of $1.5 \mathrm{~mm}$ irradiated by $\approx 113 \mathrm{mWcm}^{-2}$. This value is in the range of $50-200 \mathrm{mWcm}^{-2}$ usually chosen for ALA-induced

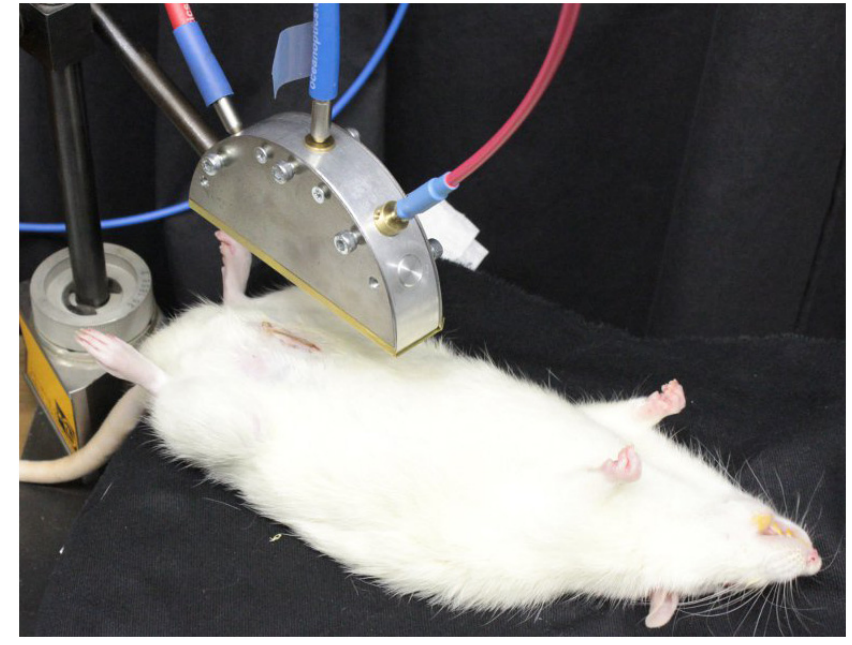

Fig. 1. The home-made holder with the three lightguides above the laboratory animal before placing on the measured tissue. The middle lightguide serves for excitation and the two other are used for collecting the fluorescence and NIR phosphorescence signal.

PpIX PDT for oncologic indications in dermatology with total light-doses ranging from 10 to $50 \mathrm{Jcm}^{-2} .^{38}$

The visible fluorescence of the PS is collected by one of the side fibers $(1 \mathrm{~mm}$ diameter VIS fiber from Ocean Optics) and spectrally resolved in fiber-optics spectrometer Avantes Ava2048. The angle between excitation and fluorescence fibers is $50^{\circ}$, which strongly reduces the specular reflection of the excitation light from the animal skin and also limits the amount of diffusely backscattered excitation light, whose intensity is the highest in the direction toward the excitation fiber and decreases with increasing angle. Moreover, an yellow long-pass filter is employed to screen the diffusely back-scattered blue excitation light in the fluorescence collecting fiber. The higher detection fiber diameter makes the elliptic spot from which the light is collected slightly larger than the excitation spot. Therefore, the emission from the whole area of the irradiated spot is collected by the fiber. The spectral sensitivity of the set-up was determined by measuring spectral density of irradiation provided by a radiometric standard. All the presented fluorescence data were corrected to the spectral sensitivity, which enables to provide absolute values of irradiation at the tip of the collecting fiber.

A home-made optical fiber with high numerical aperture of 0.48 and $1 \mathrm{~mm}$ diameter is employed to collect the NIR luminescence of the PS or that of singlet oxygen on the other side of the holder under 
$40^{\circ}$ angle to limit the collection of the backscattered light. The diameter together with higher numerical aperture of the fiber again ensures, that the elliptic area from which the luminescence is collected fully covers the whole excited spot. The full coverage of the excitation spot by both the detection fibers was checked using optics design program OSLO (Lambda Research) taking into account different materials and different wavelengths of the light for individual fibers. The remaining backscattered excitation light is screened by yellow long-pass filter (TECHSPEC $450 \mathrm{~nm}$, Edmund Optics) and the red fluorescence of the PS is screened by two RG7 long-pass filters (toptec) before entering the monochromator.

The NIR luminescence signal is fed to our homemade time- and spectral-resolved apparatus, which employs high luminosity monochromator H20 (Jobin-Yvon), NIR-sensitive photomultiplier Hamamatsu R5509, and time-resolved photon counter/multiscaler Becker Hickl MSA $200^{41}$ with 5 ns per channel. This setup, equipped by bifurcated lightguide with fluorescence probe, was successfully used to detect NIR luminescence of PSs and singlet oxygen from living mammalian cells in our previous paper. ${ }^{32}$

The holder is equipped by ports for additional fibers to enable simultaneous detection of other luminescence signals, for instance detection of timeresolved infrared phosphorescence or delayed fluorescence $^{42,43}$ of PS to obtain additional information about kinetics of the PS triplets. However, in this experiment the additional ports were sealed off due to the lack of additional detectors.

It was possible to measure between 10 to 20 individual spots in each lesion. Altogether, 170 individual spots both in lesions and in the healthy skin were investigated. This provided around 1100 fluorescence spectra and approximately 600 kinetics of infrared luminescence which made foundation for thorough statistical treatment of the obtained parameters.

\section{Results and Discussion}

PpIX synthesized in the tissues of the laboratory animals after the Metvix treatment was checked using visual observation of the PS fluorescence. Figure 2 shows the incision on the skin of the rat treated by Metvix under blue illumination through a yellow long-pass filter.

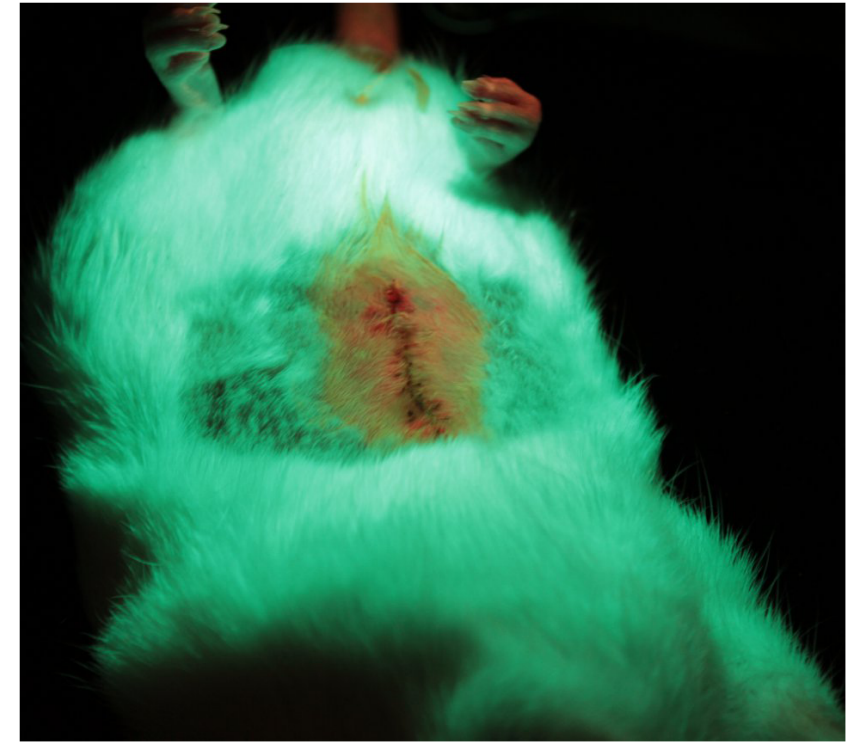

Fig. 2. The lesion on the rat after treatment by Metvix illuminated by blue light and observed through a yellow filter. The weak red luminescence around the lesion correspond to the PpIX in the healthy tissue while the bright red spots in the scar correspond to much higher concentration of the PS in the scar. The inhomogeneous fluorescence intensity in the scar reflects uneven distribution of PS in the lesion.

In the center of the shaved area on the animal's abdomen, a large weakly red-luminescent spot can be observed. It corresponds to the area of the skin, where the drug was applied and the PS was synthesized. Much brighter luminescent spots are located in the incision in the middle of the treated spot. The much higher luminescence intensity corresponds to much higher concentration of PpIX accumulated in the lesion. The fluorescence intensity is not homogeneous in the scar reflecting uneven distribution of PS in the lesion. The differences in distribution of the PS in the lesion are attributed to differences of metabolism of individual groups of cells in the healing scar (places with different nutritional transport due to damaged arteriolae and capillaries, places with local inflammation or even necrosis).

Following the visual inspection of the lesion, the fiber holder was guided to the bright spots using the fluorescence signal in the fiber-optics spectrometer. As soon as such a spot was found, time evolution of fluorescence spectra was detected in parallel to accumulation of time-resolved NIR luminescence kinetics at selected wavelengths.

The time-evolution of PpIX fluorescence spectra during the treatment of the lesion is demonstrated 


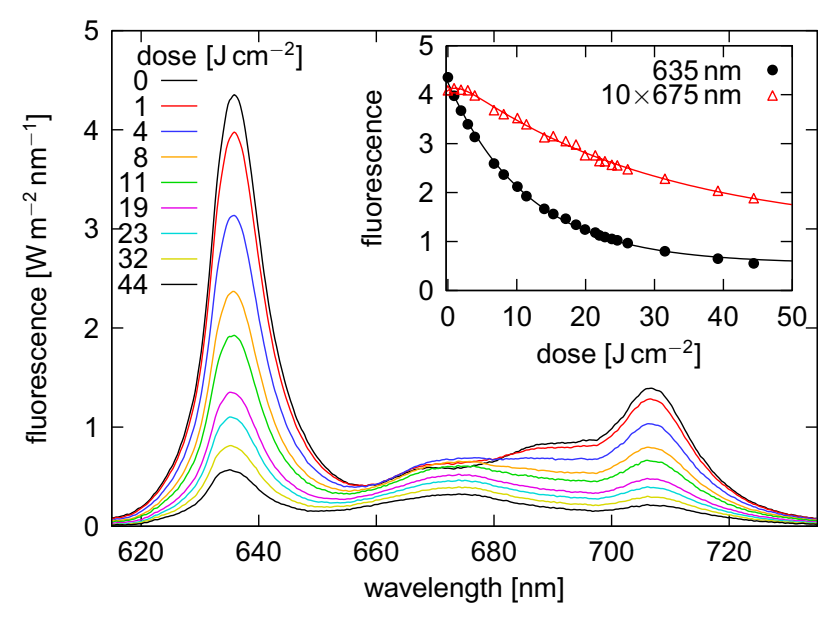

Fig. 3. Changes of fluorescence signal during exposition of the Metvix treated lesion. Inset: Bleaching of the maximum of $635 \mathrm{~nm}$ band of PpIX together with slight increase and subsequent bleaching of the photoproduct, photoprotoporphyrin IX, at $675 \mathrm{~nm}$ (10 times magnified).

in Fig. 3. The changes of intensities of maxima of fluorescence bands at 635 and $675 \mathrm{~nm}$ are documented in the inset of the figure. The decay of the fluorescence signal at $635 \mathrm{~nm}$ can be well approximated by monoexponential function. Besides gradual photobleaching of PpIX, signal around $675 \mathrm{~nm}$ slightly increases in the beginning of the treatment and then it also slowly decreases. Behavior of the band reflects formation and bleaching of the photoproduct of PpIX, Photoprotoporphyrin IX. ${ }^{9,44,45}$

Singular value decomposition (SVD) of the set of bleaching fluorescence spectra provided two principal components. The virtual spectra obtained from SVD were rotated in such a way, that the component 1 corresponds well to the fluorescence spectrum of pure PpIX, whereas the component 2 may be ascribed to the emission spectrum of the photoproducts (Fig. 4). The obtained spectrum of the photoproducts corresponds well to the spectrum of Photoprotoporphyrin, a product of reaction of ${ }^{1} \mathrm{O}_{2}$ with PpIX. ${ }^{9,44}$

Besides these two principal fluorophores, another emitting species was observed in several spots in lesion of one of the MAL-treated animals (see Fig. 5). It manifests itself as a shoulder of the $635 \mathrm{~nm}$ PpIX emission band around $622 \mathrm{~nm}$. The fluorescence signal at $622 \mathrm{~nm}$ (400 nm excitation) is used for characterization of amount of Coproporphyrin according to Ref. 46. Dietel et al. ${ }^{47}$ have assigned a fluorescence peak near $620 \mathrm{~nm}$ to the presence of Uroporphyrin and Coproporphyrin in

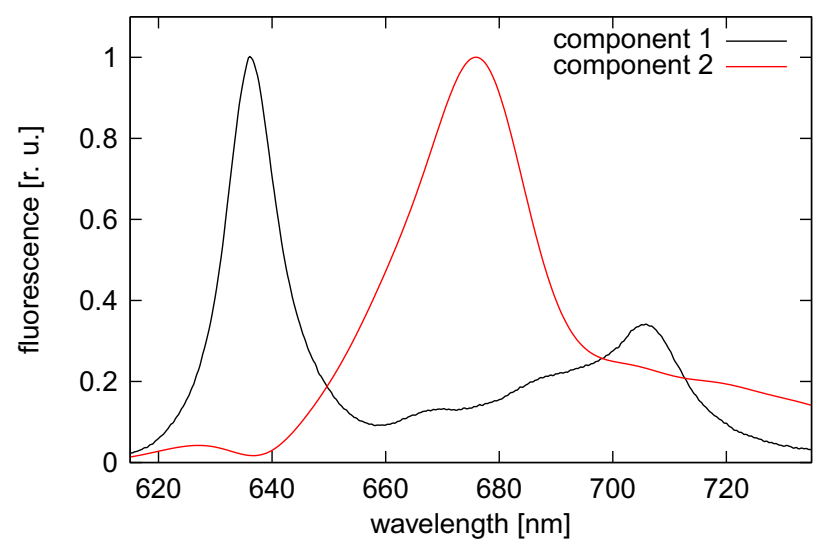

Fig. 4. Two main principal components obtained by singular decomposition of set of decaying fluorescence spectra at one selected spot in scar of an animal treated by Metvix. The component 1 corresponds well to fluorescence spectrum of pure PpIX, the component 2 may be ascribed to emission spectrum of the photoproducts.

Ehrlich ascite carcinoma cells incubated with ALA. Fluorescence peak around $620 \mathrm{~nm}$ was found by Finlay et al. in aqueous solutions of Uroporphyrin and Coproporphyrin containing lipids at physiological conditions in vitro. ${ }^{48}$ The subtraction of the two principal components obtained from SVD reveals another emission band of this species at $689 \mathrm{~nm}$ (see inset in Fig. 5). The emission spectrum of this fluorophore exhibits no detectable bleaching in the investigated range of expositions (data not shown).

Qualitatively the same behavior was observed in the lesions of the control animals, but the absolute

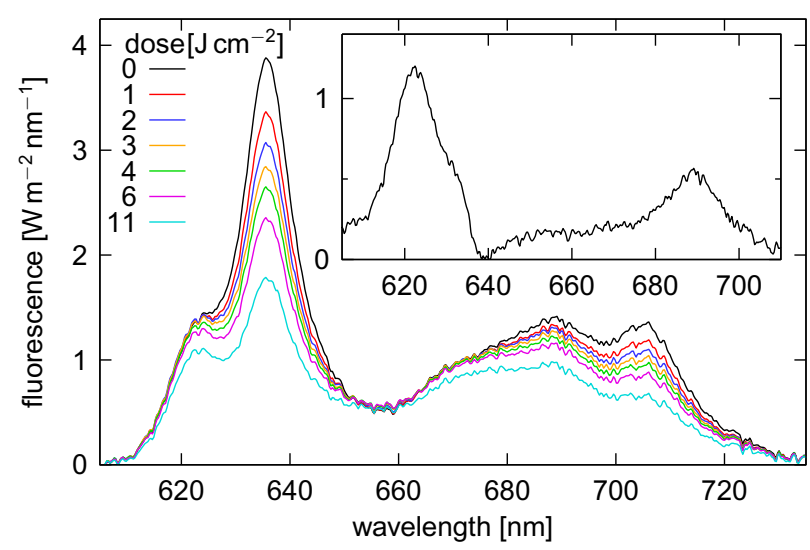

Fig. 5. Bleaching of the fluorescence of MAL-treated lesion of rat No. 6, which exhibits also contribution of luminescence of uroporphyrin and/or coproporphyrin. Inset: The emission spectrum of these two species obtained from the unbleached spectrum (dose of $0 \mathrm{Jcm}^{-2}$ ) by subtracting the two principal components obtained by SVD. 
values of the fluorescence signals in the incision of control animals were comparable to those obtained outside the lesion (see Fig. 8) which is in line with much lower concentration of naturally occurring PpIX in the incision compared to the Metvix treated one. However, Fig. 6 documents that the bleaching of the fluorescence spectra during exposition was qualitatively the same as in the MALtreated lesions.

The rate of photobleaching is described by exposition $E_{B}$ under which the intensity of the fluorescence in maximum of the emission band drops to $\frac{1}{e}$ of the initial value. The slowest photobleaching with $E_{B}=(10.0 \pm 1.3) \mathrm{J} \mathrm{cm}^{-2}$ was observed in spots in scars with PS. However, the spots in scar without PS exhibited almost the same rate of photobleaching of $(9.0 \pm 1.2) \mathrm{J} \mathrm{cm}^{2}$. Therefore, there is no statistically significant difference. Faster photobleaching was observed in spots treated by PS outside the scars with $E_{B}=(6.0 \pm 0.5) \mathrm{J} \mathrm{cm}^{-2}$. However, the difference is not statistically significant due to quite wide distributions of the values in different spots.

The rate of the photobleaching of the $635 \mathrm{~nm}$ band was in all cases faster than that of the $675 \mathrm{~nm}$. There were spots, where the latter did not bleach at all on the time scale of our experiment (data not shown). Due to the differences in the photobleaching rate between the two bands, the $675 \mathrm{~nm}$ band becomes dominant in some spots for the exposition $E_{D}$. In the others, where the fluence was not sufficient to achieve this effect, extrapolation of the photobleaching curves enables estimation of $E_{D}$. Similar values of $E_{D}$ were obtained for spots outside the scar treated by PS and spots without PS (see Table 1). Almost order of magnitude larger $E_{D}$ values were determined in spots in scars treated by PS. The effect is illustrated in Fig. 6, in which plot a) corresponds to a spot in lesion treated by MAL. The difference of $E_{D}$ is statistically significant with $p=0.04$. Therefore, determination of $E_{D}$ from

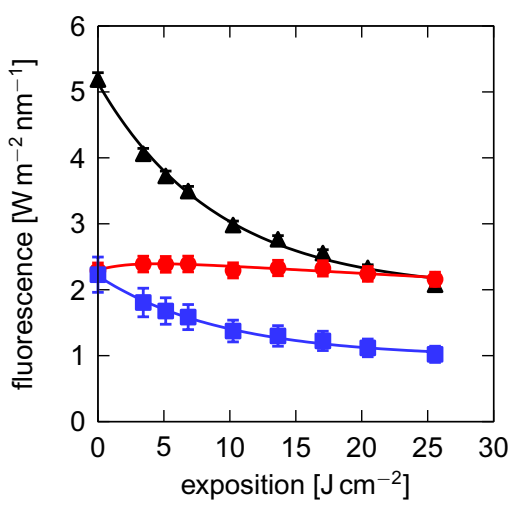

(a)

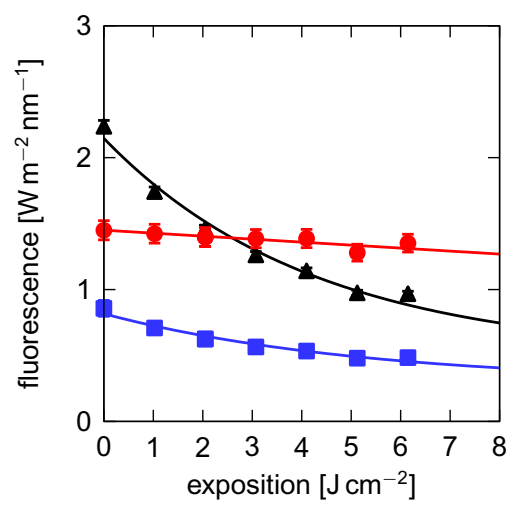

(c)

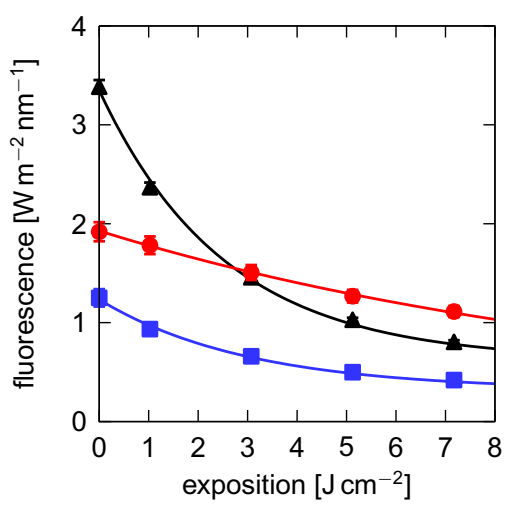

(b)

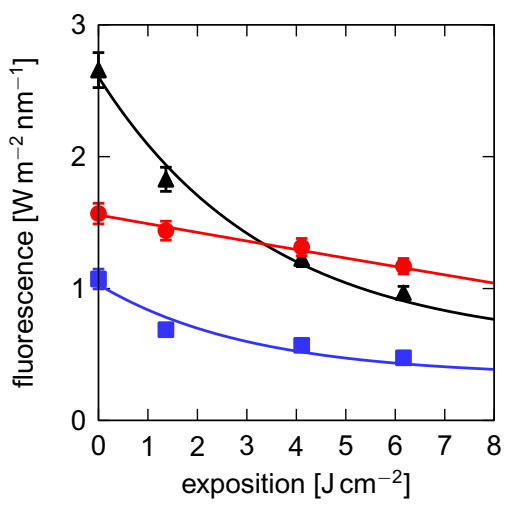

(d)

Fig. 6. Typical bleaching curves of the tree main fluorescence bands (635 nm triangles, $675 \mathrm{~nm}$ circles and $705 \mathrm{~nm}$ squares) in animals treated by MAL (top) and control (bottom) in lesion (left) and out of lesion (right). 
Table 1. Characteristic expositions for PpIX bleaching $\left(E_{B}\right)$ and dominance of the $675 \mathrm{~nm}$ spectral band $\left(E_{D}\right)$.

\begin{tabular}{lrr}
\hline Spot & \multicolumn{1}{c}{$E_{B} \mathrm{~J} \mathrm{~cm}^{-2}$} & \multicolumn{1}{c}{$E_{D} \mathrm{~J} \mathrm{~cm}^{-2}$} \\
\hline scar with PS & $10.0 \pm 1.3$ & $22.0 \pm 5.3$ \\
out of scar with PS & $6.0 \pm 0.5$ & $4.2 \pm 0.6$ \\
no PS & $9.0 \pm 1.2$ & $3.1 \pm 0.9$ \\
\hline
\end{tabular}

differences in bleaching of fluorescence at 635 and $675 \mathrm{~nm}$ offers a potential measure for photodiagnostic applications.

Figure 7 shows phosphorescence kinetics of PpIX and ${ }^{1} \mathrm{O}_{2}$. The PpIX and ${ }^{1} \mathrm{O}_{2}$ phosphorescence kinetics are collected on the same experimental animal, however, at different spots in the incision due to the lack of the second phosphorescence channel which would enable simultaneous detection. A sequential detection of the kinetics is not applicable due to the bleaching of the spots. Nevertheless, the differences of phosphorescence kinetics in different spots in the lesion are such small that it makes comparison of the two phosphorescence kinetics possible. The PpIX phosphorescence follows complex time evolution which was approximated by linear combination of three exponential decays with lifetimes of $(0.42 \pm 0.05),(2.6 \pm 0.1)$ and $(40 \pm 5) \mu \mathrm{s}$. Such a complicated behavior was observed in our previous experiments on living cells. ${ }^{32}$ It reflects heterogeneous environment of different groups of PS in different locations in the cells. The heterogeneity of environment causes differences in the efficiency of quenching of PS triplets by oxygen, analogous to our earlier studies of two groups of

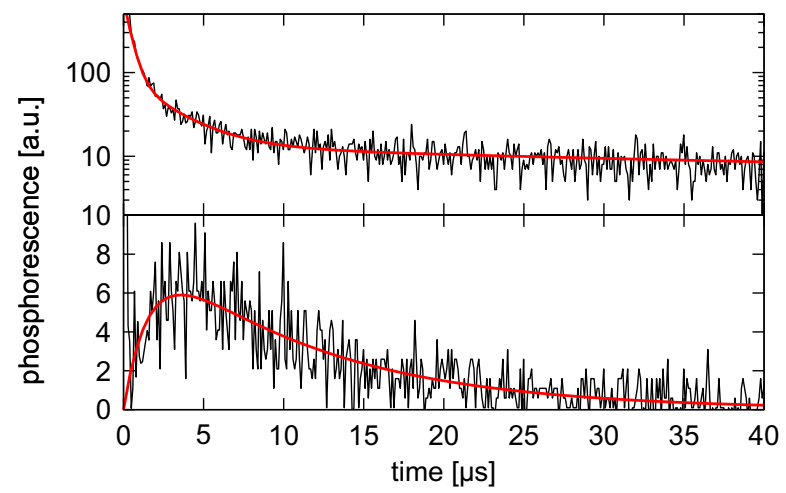

Fig. 7. Typical phosphorescence kinetics of PpIX at $910 \mathrm{~nm}$ approximated by combination of three exponential decays (top) and singlet oxygen at $1278 \mathrm{~nm}$ approximated by combination of single-exponential rise and decay (bottom).
PpIX in liposomes quenched by oxygen with different efficiencies. ${ }^{23,24}$ It is reflected in the presence of multiple decay components in PS phosphorescence. The compound character of the PpIX decay suggests that the decay of ${ }^{1} \mathrm{O}_{2}$ should follow similarly complex time development. Nevertheless, the kinetics of ${ }^{1} \mathrm{O}_{2}$ can be approximated by plain convolution of two exponentials with lifetimes of $(1.6 \pm 0.2)$ and $(10.7 \pm 0.5) \mu$ s quite well. More complex models were also tested. However, such fits were less reliable than the simple model. Unfortunately, neither the rise nor the decay component of ${ }^{1} \mathrm{O}_{2}$ kinetics of $(10.7 \pm 0.5) \mu$ s corresponds to a single lifetime in the PpIX kinetics. This effect was already reported in 3T3 mouse fibroblasts with ALA-induced PpIX in vitro. ${ }^{32}$ The discrepancy may originate in the fact, that the molecules quenched less efficiently by oxygen are more pronounced in PpIX emission kinetics. On the contrary, their contribution to the ${ }^{1} \mathrm{O}_{2}$ kinetics is weaker. Therefore, the effective lifetimes observed in both kinetics differ. The role of effective value is supported by fact, that it lies between the lifetimes of the longer components of PpIX decay.

The lack of a component in PpIX phosphorescence kinetics exhibiting the same lifetime as one of the time-constants of ${ }^{1} \mathrm{O}_{2}$ kinetics makes assignment of the time-constants to lifetimes of ${ }^{1} \mathrm{O}_{2}$ and PpIX triplets difficult. On one hand, a number of studies on cell cultures report dramatically shortened lifetime of ${ }^{1} \mathrm{O}_{2}$ in cellular environment compared to water solutions due to faster quenching by biological molecules. For instance, there were published ${ }^{1} \mathrm{O}_{2}$ lifetimes around $0.6 \mu \mathrm{s}$ in AML or P388 leukemia cells with $\mathrm{AlPcS}_{4}$ (aluminum phtalocyanine tetrasulfonate), ${ }^{1,18} 1.7 \mu \mathrm{s}$ in human skin fibroblasts with TMPyP $(5,10,15,20$-tetrakis(N-methyl-4-pyridyl)-porphine) and $\mathrm{TPPS}_{4}(5,10,15,20$-tetrakis(4sulphonatophenyl) porphine, ${ }^{25}$ or $1.4-2.0 \mu \mathrm{s}$ in Staphylococcus aureus or E. coli bacterial suspensions with TMPyP. ${ }^{49}$ The results were obtained on hydrophilic PSs which localize inside lysosomes $\left(\mathrm{AlPcS}_{4}\right.$ and $\left.\mathrm{TPPS}_{4}\right)$ or nuclei of the cells (TMPyP). ${ }^{25,32}$ Similar ${ }^{1} \mathrm{O}_{2}$ lifetime of $0.6 \mu$ s was identified also in in vitro ALA-sensitized AML5 leukemia cells. ${ }^{14}$ Authors of this paper have investigated ${ }^{1} \mathrm{O}_{2}$ kinetics in in vitro $3 \mathrm{~T} 3$ mouse fibroblast suspensions with $\mathrm{TPPS}_{4}, \mathrm{TMPyP}$ and lipophilic ALA-induced PpIX using the same experimental set-up as has been used in this study. ${ }^{32}$ ALA-induced PpIX localizes in mitochondria of the cells in 
contrast to the externally applied one, which localizes in cell membranes. The ${ }^{1} \mathrm{O}_{2}$ lifetimes of $0.8 \mu \mathrm{s}$ obtained in the study matched perfectly those obtained using $\mathrm{TPPS}_{4}$. All these evidence supports assignment of the shorter time-constant to the ${ }^{1} \mathrm{O}_{2}$ lifetime. On the other hand, a number of studies carried on ex vivo porcine skin unequivocally identify the longer time-constant of ${ }^{1} \mathrm{O}_{2}$ kinetics with its lifetime using external quenchers or changes in oxygen partial pressure. For example, 12.5-19.2 $\mu \mathrm{s}$ obtained with pheophorbide-a ${ }^{13,50}$ or $18 \mu$ s with $1 \mathrm{H}-$ phenalen-1-one or 5,10,15,20-tetra phenyl porphine (TPP). ${ }^{51}$ In both cases, externally applied lipophilic PSs were used. Exogenous lipophilic PSs usually localize in cell membranes. Therefore, the lifetime of ${ }^{1} \mathrm{O}_{2}$ can be compared to those obtained in lipids (6$14 \mu \mathrm{s}$ in phosphatidylcholine $\left.{ }^{21,52}\right)$ or in liposomes ( $7 \mu$ s for TPP in phosphatidylcholine unilamellar liposomes $\left.{ }^{23,24}\right)$. It must be added, that $8 \mu$ s decay component in ${ }^{1} \mathrm{O}_{2}$ phosphorescence was observed under ultraviolet (UVA) excitation of endogenous PSs in porcine skin ex vivo, however, direct assignment of this component to ${ }^{1} \mathrm{O}_{2}$ or PS triplet states was not provided. ${ }^{53}$ Unfortunately, the data presented in this paper are not able to assign the ${ }^{1} \mathrm{O}_{2}$ kinetic components unequivocally. However, in our opinion, behavior of the ${ }^{1} \mathrm{O}_{2}$ kinetics described later in the text suggest identification of the shorter timeconstant with lifetime of ${ }^{1} \mathrm{O}_{2}$.

The $\approx(11 \pm 5) \mu$ s decays obtained in our study are slightly shorter than $(16 \pm 2) \mu$ s obtained earlier by Jarvi et al. ${ }^{18}$ and $13-19 \mu$ s presented by Schlothauer et al. ${ }^{13}$ The ${ }^{1} \mathrm{O}_{2}$ rise times of $1.3-1.6 \mu$ s are shorter than $(2.4 \pm 0.5) \mu$ s published in Ref. 18 but faster than $0.3-0.9 \mu$ s reported in Ref. 13. However, the rise-components of ${ }^{1} \mathrm{O}_{2}$ in the latter study may be influenced by the fact, that first $1.6 \mu$ s of the kinetics were excluded from the fitting due to overlap with signal artifacts. Compared to our previous study on living 3T3 mouse fibroblasts with endogenous PpIX, ${ }^{32}$ both time-constants of ${ }^{1} \mathrm{O}_{2}$ kinetics are longer than $(0.8 \pm 0.1)$ and $(6.2 \pm 0.4) \mu$ s obtained in cultured cells. It can be explained by higher concentration of dissolved oxygen in cell suspension compared to the tissues of living animals.

Figure 8 presents the relationship between maximal fluorescence signal at $635 \mathrm{~nm}$ and ${ }^{1} \mathrm{O}_{2}$ luminescence intensity integrated over the whole decay at individual spots during initial exposition. Unfortunately, there is no straight correlation between these two features with Pearson's correlation

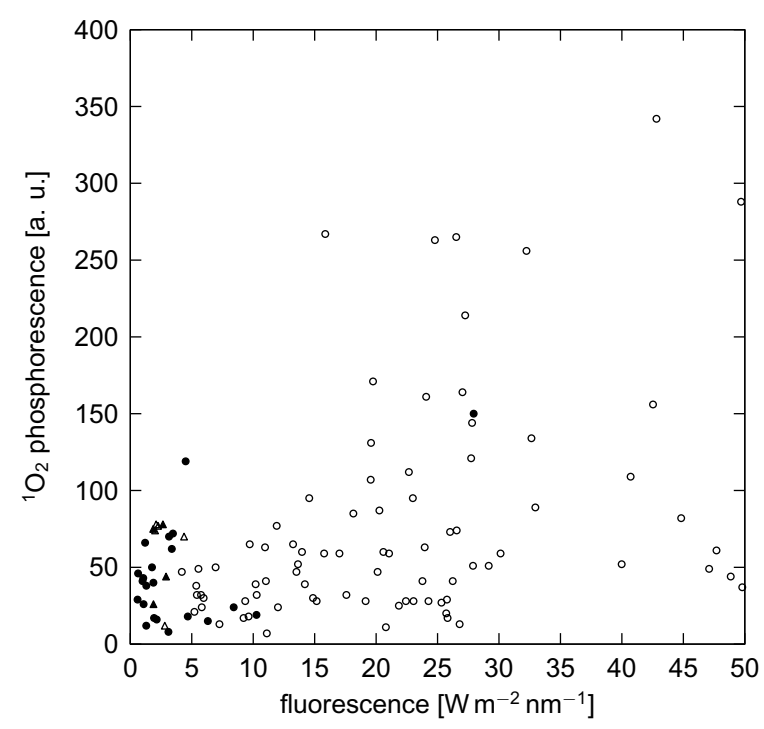

Fig. 8. The lack of direct correlation between the maximal PpIX fluorescence signal at $635 \mathrm{~nm}$ and the total intensity of ${ }^{1} \mathrm{O}_{2}$ luminescence at the initial exposition at individual spots is documented by abundance of points with high fluorescence but low ${ }^{1} \mathrm{O}_{2}$ phosphorescence appearing in the lower right part of the plot. Circles represent MAL-treated animals whereas triangles correspond to the control ones in lesion (open symbols) as well as outside of it (full symbols).

coefficient of $r=0.4$. It is best documented by the fact, that there are numerous spots exhibiting very strong signal of PpIX fluorescence and only weak ${ }^{1} \mathrm{O}_{2}$ phosphorescence (lower right part of the plot). Therefore, high PpIX fluorescence signal seems to be a necessary but not a sufficient condition for obtaining strong ${ }^{1} \mathrm{O}_{2}$ luminescence. This is in contrast with linear correlation between PpIX fluorescence and singlet oxygen signal in ALA treated healthy human skin reported by Mallidi et al. ${ }^{10}$ The discrepancy is probably caused by higher heterogeneity of the scar tissue compared to the human skin samples. It results in presence of high amounts of PpIX which is not able to transfer energy to singlet oxygen efficiently in particular spots in the lesions. This fact makes utilization of the PpIX fluorescence signal for dosimetry of singlet oxygen production in in vivo systems questionable.

Moreover, neither the rate of photobleaching of PpIX fluorescence $\left(E_{B}\right)$ nor the exposition when the photoproduct fluorescence band becomes dominant $\left(E_{D}\right)$ correlate with ${ }^{1} \mathrm{O}_{2}$ luminescence intensity well with $r=0.2$ and $r=0.3$, respectively (data not shown). Nevertheless, a separated treatment of the spots in and outside of the lesion provides better correlation between the total intensity of ${ }^{1} \mathrm{O}_{2}$ 


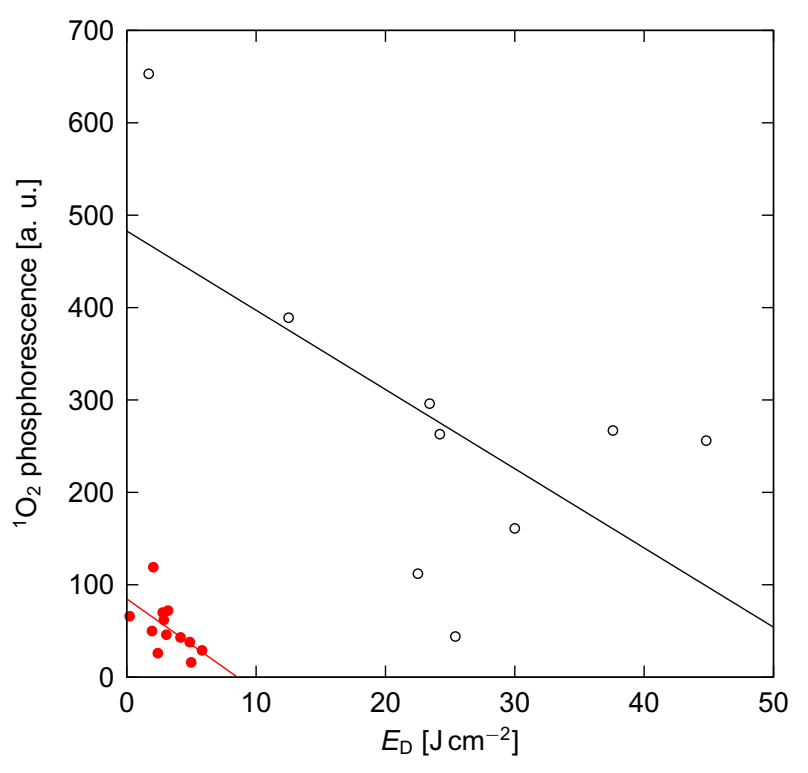

Fig. 9. The correlation between the total intensity of ${ }^{1} \mathrm{O}_{2}$ luminescence at the initial exposition with exposition when the photoproduct fluorescence band becomes dominant $\left(E_{D}\right)$ at individual spots in lesion (open circles) and normal skin (full circles) of MAL-treated animals accompanied by linear approximations of the dependencies.

luminescence and $E_{D}$ value with Pearson's coefficients of $r=-0.6$ for both sets of values (see Fig. 9). However, the figure documents, that the dependence is probably not linear. Unfortunately, it is not possible to speculate about the mechanism that should provide a better fitting model. Anyway, the correlation conforms with the idea that the higher production of ${ }^{1} \mathrm{O}_{2}$ correlates with lower exposition needed to reach the dominance of the photoproduct in PS fluorescence spectrum due to faster production of the photoproduct. However, similar values of ${ }^{1} \mathrm{O}_{2}$ luminescence provide different $E_{D}$ expositions in spots in and outside of the lesion. No such correlation was found in the case of PpIX bleaching rate expressed by $E_{B}$ value. That is probably caused by different influence of non- ${ }^{1} \mathrm{O}_{2}$ mediated PpIX photobleaching mechanisms ${ }^{7,9}$ in different spots of the inhomogeneous scar tissues.

The total intensity of ${ }^{1} \mathrm{O}_{2}$ luminescence (i.e., integrated over the whole decay) decreases during the light treatment similarly to the fluorescence of PpIX in the Metvix treated animals (see Fig. 10). The decrease reflects drop in ${ }^{1} \mathrm{O}_{2}$ concentration which may be caused either by decreasing concentration of the PS (observed by decrease of its fluorescence) or by decrease of the oxygen concentration in the treated tissue due to light-induced oxidation

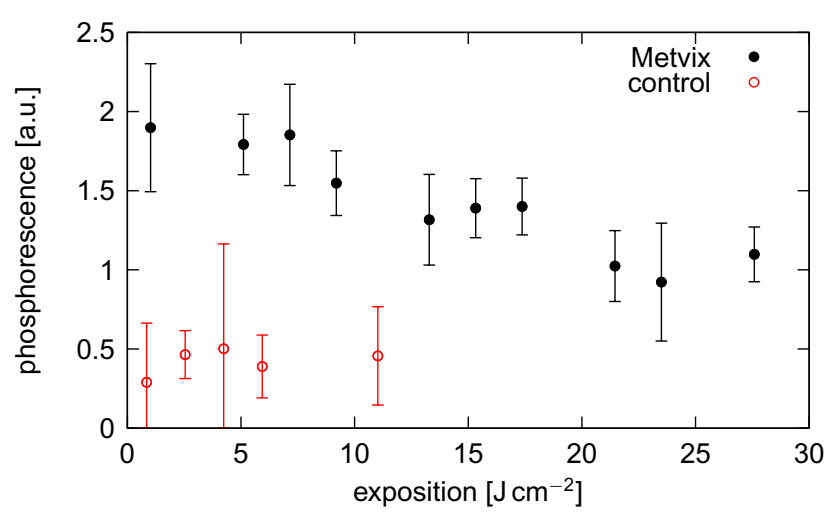

Fig. 10. Singlet oxygen phosphorescence intensity slowly drops with exposition of the lesion in Metvix treated animals (solid) while it remains unchanged in the control (open) ones during the treatment.

reactions. If the decay time-constant of ${ }^{1} \mathrm{O}_{2}$ kinetics corresponds to PpIX triplet lifetimes, the latter phenomenon is in line also with slight increase of the lifetimes of the PS (displayed in Fig. 11) which may be caused by lower quenching of the PS triplets by oxygen. Moreover, the effect of increased intracellular viscosity in response to photodynamic treatment ${ }^{54}$ may also lead to weaker quenching and thus reduced ${ }^{1} \mathrm{O}_{2}$ production. Taking into account the hypothesis that the decay time-constants represent ${ }^{1} \mathrm{O}_{2}$ lifetime, the increase can be explained by lower quenching of ${ }^{1} \mathrm{O}_{2}$ due to depletion of its reaction targets by oxidative reactions. Similar effect was reported for instance in reactions of singlet oxygen with human serum albumin (HSA) in $\mathrm{TPPS}_{4}$ solutions. $^{19}$ Taking into account the abundance of oxidation targets in cellular environment, this explanation seems less probable, supporting the

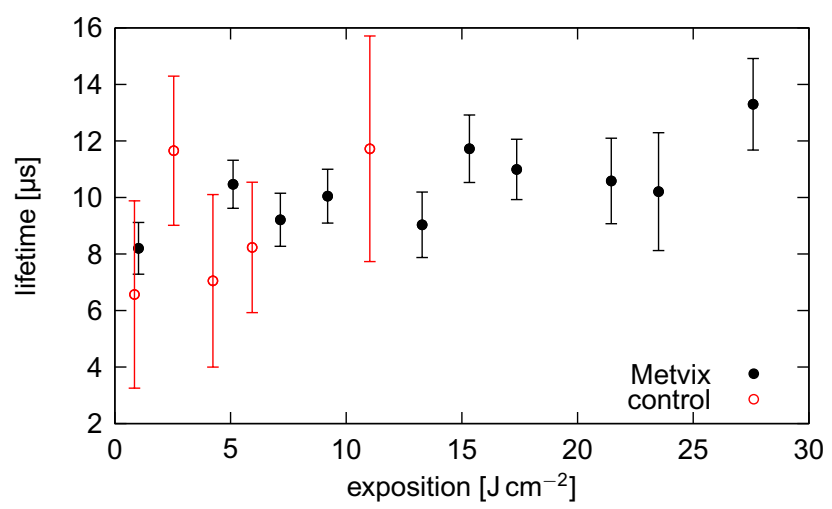

Fig. 11. Singlet oxygen phosphorescence decay times increase during the treatment both in Metvix treated (solid) and control (open) animals. 


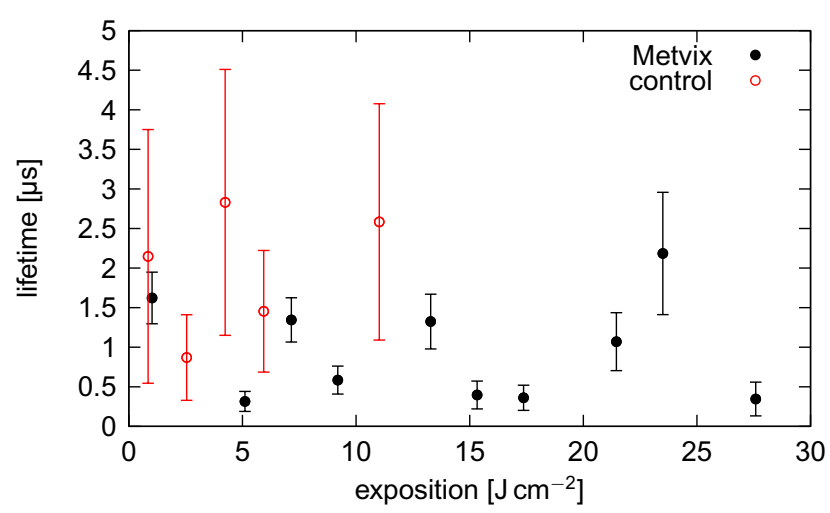

Fig. 12. Singlet oxygen phosphorescence rise times exhibit exposition dependence neither in Metvix treated (solid) nor in control (open) animals.

identification of the longer time-constant with PpIX triplet lifetime.

Despite the fact that PpIX fluorescence bleaches also in the control animals, no matching decrease of total ${ }^{1} \mathrm{O}_{2}$ luminescence intensity was observed (Fig. 10). Substantially lower amounts of singlet oxygen are photogenerated in the control animals. It is reflected in much lower ${ }^{1} \mathrm{O}_{2}$ phosphorescence intensities. Lower amounts of ${ }^{1} \mathrm{O}_{2}$ imply also slower PDT-induced depletion of oxygen in the treated tissues. However, the increasing trend in lifetimes of PpIX triplets obtained from ${ }^{1} \mathrm{O}_{2}$ decays (Fig. 11) does not fit in this line.

The exposition dependence of ${ }^{1} \mathrm{O}_{2}$ rise-times was examined as well. The rise-times of ${ }^{1} \mathrm{O}_{2}$ plotted in Fig. 12 exhibit dependence on exposition of the tissue during the irradiation neither for Metvix treated nor for control animals. However, the rise-times in control animals are slightly higher. This effect can be explained in the case of the rise-time corresponding to the lifetime of ${ }^{1} \mathrm{O}_{2}$ due to lower quenching of ${ }^{1} \mathrm{O}_{2}$ by either PpIX or ${ }^{1} \mathrm{O}_{2}$ itself. Under assumption that the rise-times correspond to the PpIX lifetimes, lower quenching of PpIX by oxygen would explain this phenomenon. However, there is no reason for such a lower quenching in the control group.

The statistic analysis of variance revealed a statistically significant difference in lifetimes of ${ }^{1} \mathrm{O}_{2} \mathrm{lu}$ minescence between spots in and outside the incisions with $p=0.05$ but no significant correlations between spots treated and untreated by MAL $(p=0.31)$. On the contrary, the difference in PpIX triplet luminescence decay times may exhibit statistical difference with null hypothesis probability $p=0.07$ between spots treated and untreated by MAL, whereas no significant difference was obtained between spots in and outside the lesions $(p=0.67)$.

\section{Conclusions}

The results show that the sensitivity of the used setup is sufficient to obtain time-resolved kinetics of weak infrared singlet oxygen luminescence from cutaneous lesions treated by MAL in laboratory animals in vivo during photodynamic treatment. The usage of optical lightguides for collection of the emission signals provides easy access to different spots of the lesions bringing significant advantage for potential clinical application, possibly even in combination with endoscopic equipment. Moreover, the employment of lightguides opens possibility of simultaneous detection of additional emission signals, e.g., spectrally resolved visible fluorescence of the PS, which was also demonstrated. The simultaneous detection of fluorescence enables not only guiding of the device to the lesion, but also fluorescence diagnosis and implicit PDT dosimetry. However, there was found no proportionality between fluorescence intensity and production of singlet oxygen. Anyway, strong signal of PSs fluorescence was proved to be a necessary but not a sufficient condition for higher singlet oxygen production under in vivo conditions. On the contrary, the rate of production of the PS photoproduct represented as a exposition under which the photoproduct emission band becomes dominant feature in the fluorescence spectrum was shown to be a good indicator for differentiation between normal skin and lesion treated by MAL. Moreover, this parameter correlates rather well with production of singlet oxygen. A statistically significant correlation between spots outside and in the lesion were found also for rise-times of singlet oxygen kinetics corresponding to the lifetimes of singlet oxygen itself with no correlation to the MAL treatment of the spot. In contrast, the lifetimes of PS triplets reflected in decay-times of singlet oxygen luminescence exhibit weak difference between spots treated and untreated by MAL, whereas no difference was found between spots in and outside of the lesion.

\section{Acknowledgments}

This work was supported by the Charles University research programs PRVOUK P34 and P45 and $260045 / \mathrm{SVV} / 2014$. 


\section{References}

1. M. Niedre, M. S. Patterson, B. C. Wilson, "Direct near-infrared luminescence detection of singlet oxygen generated by photodynamic therapy in cells in vitro and tissues in vivo," Photochem. Photobiol. 75, 382-391 (2002).

2. R. L. van Veen, D. J. Robinson, P. D. Siersema, H. J. Sterenborg, "The importance of in situ dosimetry during photodynamic therapy of barrett's esophagus," Gastrointest. Endosc. 64, 786-788 (2006).

3. B. W. Henderson, T. M. Busch, J. W. Snyder, "Fluence rate as a modulator of PDT mechanisms," Lasers Surg. Med. 38, 489-493 (2006).

4. B. Wilson, M. Patterson, L. Lilge, "Implicit and explicit dosimetry in photodynamic therapy: a new paradigm," Lasers Med. Sci. 12, 182-199 (1997).

5. B. C. Wilson, M. S. Patterson, "The physics, biophysics and technology of photodynamic therapy," Phys. Med. Biol. 53, R61-R109 (2008).

6. M. A. Weston, M. S. Patterson, "Monitoring oxygen concentration during photodynamic therapy using prompt photosensitizer fluorescence," Phys. Med. Biol. 58, 7039-7059 (2013).

7. I. Georgakoudi, T. H. Foster, "Singlet oxygenversus nonsinglet oxygen-mediated mechanisms of sensitizer photobleaching and effects on photodynamic dosimetry," Photochem. Photobiol. 67, 612625 (1998).

8. J. C. Finlay, S. Mitra, M. S. Patterson, T. H. Foster, "Photobleaching kinetics of photofrin in vivo and in multicell tumour spheroids indicate two simultaneous bleaching mechanisms," Phys. Med. Biol. 49, 4837 (2004).

9. J. S. Dysart and M. S. Patterson, "Photobleaching kinetics, photoproduct formation, and dose estimation during ALA induced PpIX PDT of MLL cells under well oxygenated and hypoxic conditions," Photochem. Photobiol. Sci. 5, 73-81 (2006).

10. S. Mallidi, S. Anbil, S. Lee, D. Manstein, S. Elrington, G. Kositratna, D. Schoenfeld, B. Pogue, S. J. Davis, T. Hasan, "Photosensitizer fluorescence and singlet oxygen luminescence as dosimetric predictors of topical 5-aminolevulinic acid photodynamic therapy induced clinical erythema," J. Biomed. Opt. 19, 028001 (2014).

11. M. T. Jarvi, M. S. Patterson, B. C. Wilson, "Insights into photodynamic therapy dosimetry: Simultaneous singlet oxygen luminescence and photosensitizer photobleaching measurements," Biophys. J. 102, 661-671 (2012).
12. B. Li, H. Lin, D. Chen, B. C. Wilson, Y. Gu, "Singlet oxygen detection during photosensitization," J. Innov. Opt. Health Sci. 6, 1330002 (2013).

13. J. C. Schlothauer, J. Falckenhayn, T. Perna, S. Hackbarth, B. Röder, "Luminescence investigation of photosensitizer distribution in skin: Correlation of singlet oxygen kinetics with the microarchitecture of the epidermis," J. Biomed. Opt. 18, 115001 (2013).

14. M. J. Niedre, A. J. Secord, M. S. Patterson, B. C. Wilson, "In vitro tests of the validity of singlet oxygen luminescence measurements as a dose metric in photodynamic therapy," Cancer Res. 63, 79867994 (2003).

15. M. J. Niedre, C. S. Yu, M. S. Patterson, B. C. Wilson, "Singlet oxygen luminescence as an in vivo photodynamic therapy dose metric: Validation in normal mouse skin with topical amino-levulinic acid," Br. J. Cancer 92, 289-304 (2005).

16. B. C. Wilson, M. S. Patterson, B. Li, M. T. Jarvi, "Correlation of in vivo tumor response and singlet oxygen luminescence detection in mTHPC-mediated photodynamic therapy," J. Innov. Opt. Health Sci. 8, 1540006 (2015).

17. M. Niedre, M. S. Patterson, A. Giles, B. C. Wilson, "Imaging of photodynamically generated singlet oxygen luminescence in vivo," Photochem. Photobiol. 81, 941-943 (2005).

18. M. T. Jarvi, M. J. Niedre, M. S. Patterson, B. C. Wilson, "Singlet oxygen luminescence dosimetry (SOLD) for photodynamic therapy: Current status, challenges and future prospects," Photochem. Photobiol. 82, 1198-1210 (2006).

19. M. Kořínek, R. Dědic, A. Molnár, J. Hála, "The influence of human serum albumin on the photogeneration of singlet oxygen by meso-tetra(4-sulfonatophenyl)porphyrin. An infrared phosphorescence study," J. Fluoresc. 16, 355-359 (2006).

20. P. Gbur, R. Dědic, D. Chorvát, Jr., P. Miškovský, J. Hála, D. Jancura, "Time resolved luminescence and singlet oxygen formation after illumination of hypericin-low-density lipoproteins complex," Photochem. Photobiol. 85, 816-823 (2009).

21. R. Dědic, V. Vyklický, A. Svoboda, J. Hála, "Singlet oxygen lifetime dependence on photosensitizer concentration in lipid films," J. Lumines. 131, 442-444 (2011).

22. R. Dědic, A. Molnár, A. Svoboda, J. Hála, "Lightinduced TPP photoproduct formation in chloroform and protective role of lipids," J. Porphyr. Phthalocyanines 14, 962-967 (2010).

23. A. Molnár, R. Dědic, A. Svoboda, J. Hála, "Singlet oxygen production by lipophilic photosensitizers in liposomes studied by time and spectral resolved 
phosphorescence," J. Mol. Struct. 834, 488-491 (2007).

24. A. Molnár, R. Dědic, A. Svoboda, J. Hála, "Spectroscopic study of singlet oxygen photogeneration by lipophilic photosensitizer in liposomes," $J$. Lumines. 128, 783-785 (2008).

25. A. Jiménez-Banzo, M. L. Sagristà, M. Mora, S. Nonell, "Kinetics of singlet oxygen photosensitization in human skin fibroblasts," Free Radic. Biol. Med. 44, 1926-1934 (2008).

26. S. Lee, L. Zhu, A. M. Minhaj, M. F. Hinds, D. H. Vu, D. I. Rosen, S. J. Davis, T. Hasan, "Pulsed diode laser-based monitor for singlet molecular oxygen," J. Biomed. Opt. 13, 034010 (2008).

27. J. Schlothauer, S. Hackbarth, B. Röder, "A new benchmark for time-resolved detection of singlet oxygen luminescence - revealing the evolution of lifetime in living cells with low dose illumination," Laser Phys. Lett. 6, 216-221 (2009).

28. S. Hackbarth, J. Schlothauer, A. Preuss, B. Röder, "New insights to primary photodynamic effects singlet oxygen kinetics in living cells," J. Photochem. Photobiol. B-Biol. 98, 173-179 (2010).

29. M. T. Jarvi, M. J. Niedre, M. S. Patterson, B. C. Wilson, "The influence of oxygen depletion and photosensitizer triplet-state dynamics during photodynamic therapy on accurate singlet oxygen luminescence monitoring and analysis of treatment dose response," Photochem. Photobiol. 87, 223-234 (2011).

30. S. Hatz, L. Poulsen, P. R. Ogilby, "Time-resolved singlet oxygen phosphorescence measurements from photosensitized experiments in single cells: Effects of oxygen diffusion and oxygen concentration," Photochem. Photobiol. 84, 1284-1290 (2008).

31. P. R. Ogilby, "Singlet oxygen: There is indeed something new under the sun," Chem. Soc. Rev. 39, 3181-3209 (2010).

32. V. Vyklický, R. Dědic, N. Curkaniuk, J. Hála, "Spectral- and time-resolved phosphorescence of photosensitizers and singlet oxygen: From in vitro towards in vivo," J. Lumines. 143, 729-733 (2013).

33. J. Hegyi, V. Hegyi, T. Ruzicka, P. Arenberger, C. Berking, "New developments in fluorescence diagnostics," J. Dtsch. Dermatol. Ges. 9, 368-372 (2011).

34. M. S. Eljamel, "Fluorescence image-guided surgery of brain tumors: Explained step-by-step," Photodiagnosis Photodyn. Ther. 5, 260-263 (2008).

35. M. Kriz, J. Hegyi, T. Ruzicka, C. Berking, "Fluorescence diagnostics as a guide for demarcation and biopsy of suspected anal cancer," Int. J. Dermatol. 51, 31-34 (2012).

36. B. Krammer, K. Plaetzer, "ALA and its clinical impact, from bench to bedside," Photochem. Photobiol. Sci. 7, 283-289 (2008).
37. B. Nokes, M. Apel, C. Jones, G. Brown, J. E. Lang, "Aminolevulinic acid (ALA): Photodynamic detection and potential therapeutic applications," $J$. Surg. Res. 181, 262-271 (2013).

38. R. Pottler, B. Krammer, R. Baumgartner, H. Stepp, editors, Photodynamic Therapy with ALA. A Clinical Handbook, volume 6 of Comprehensive Series in Photochemistry and Photobiology, RSC Publishing, Cambridge (2006).

39. M. Horn, P. Wolf, H. Wulf, T. Warloe, C. Fritsch, L. Rhodes, R. Kaufmann, M. De Rie, F. Legat, I. Stender, A. Solér, A.-M. Wennberg, G. Wong, O. Larkö, "Topical methyl aminolaevulinate photodynamic therapy in patients with basal cell carcinoma prone to complications and poor cosmetic outcome with conventional treatment," $\mathrm{Br}$. J. Dermatol. 149, 1242-1249 (2003).

40. P. Foley, M. Freeman, A. Menter, G. Siller, R. A. El-Azhary, K. Gebauer, N. J. Lowe, M. T. Jarratt, D. F. Murrell, P. Rich, D. M. Pariser, A. R. Oseroff, R. Barnetson, C. Anderson, S. Kossard, L. E. Gibson, W. D. Tope, "Photodynamic therapy with methyl aminolevulinate for primary nodular basal cell carcinoma: Results of two randomized studies," Int. J. Dermatol. 48, 1236-1245 (2009).

41. R. Dědic, A. Svoboda, J. Pšenčík, J. Hála, "Phosphorescence of singlet oxygen and meso-tetra(4sulfonatophenyl)porphin: Time and spectral resolved study," J. Mol. Struct. 651-653, 301-304 (2003).

42. M. Scholz, R. Dědic, J. Hála, S. Nonell, "Oxygen effects on tetrapropylporphycene near-infrared luminescence kinetics," J. Mol. Struct. 1044, 303-307 (2013).

43. M. Scholz, R. Dědic, T. Breitenbach, J. Hála, "Singlet oxygen-sensitized delayed fluorescence of common water-soluble photosensitizers," Photochem. Photobiol. Sci. 12, 1873-1884 (2013).

44. S. Bagdonas, L.-W. Ma, V. Iani, R. Rotomskis, P. Juzenas, J. Moan, "Phototransformations of 5-aminolevulinic acid-induced protoporphyrin IX in vitro: A spectroscopic study," Photochem. Photobiol. 72, 186-192 (2000).

45. P. Juzenas, V. Iani, S. Bagdonas, R. Rotomskis, J. Moan, "Fluorescence spectroscopy of normal mouse skin exposed to 5-aminolaevulinic acid and red light," J. Photochem. Photobiol. B-Biol. 61, 78-86 (2001).

46. C. A. Rebeiz, "Analysis of intermediates and end products of the chlorophyll biosynthestic pathway," Heme, Chlorophyll, and Bilins: Methods and Protocols, A. Smith and M. Witty, Eds., pp. 111-156, Humana Press (2002).

47. W. Dietel, K. Bolsen, E. Dickson, C. Fritsch, R. Pottier, R. Wendenburg, "Formation of water-soluble porphyrins and protoporphyrin $\{\mathrm{IX}\}$ in 
5-aminolevulinic-acid-incubated carcinoma cells," J. Photochem. Photobiol. B-Biol. 33, 225-231 (1996).

48. J. C. Finlay, D. L. Conover, E. L. Hull, T. H. Foster, "Porphyrin bleaching and PDT-induced spectral changes are irradiance dependent in ALA-sensitized normal rat skin in vivo," Photochem. Photobiol. 73, 54-63 (2001).

49. J. Regensburger, T. Maisch, A. Felgenträger, F. Santarelli, W. Bäumler, "A helpful technology the luminescence detection of singlet oxygen to investigate photodynamic inactivation of bacteria (PDIB)," J. Biophotonics 3, 319-327 (2010).

50. J. C. Schlothauer, S. Hackbarth, L. Jäger, K. Drobniewski, H. Patel, S. M. Gorun, B. Röder, "Time-resolved singlet oxygen luminescence detection under photodynamic therapy relevant conditions: Comparison of ex vivo application of two photosensitizer formulations," J. Biomed. Opt. 17, 115005 (2012).
51. S. Nonell, M. García-Díaz, J. L. Viladot, R. Delgado, "Singlet molecular oxygen quenching by the antioxidant dimethylmethoxy chromanol in solution and in ex vivo porcine skin," Int. J. Cosmetic Sci. 35, 272-280 (2013).

52. J. Baier, M. Maier, R. Engl, M. Landthaler, W. Bäumler, "Time-resolved investigations of singlet oxygen luminescence in water, in phosphatidylcholine, and in aqueous suspensions of phosphatidylcholine or HT29 cells," J. Phys. Chem. B 109, 3041-3046 (2005).

53. J. Baier, T. Maisch, M. Maier, M. Landthaler, W. Bäumler1, "Direct detection of singlet oxygen generated by UVA irradiation in human cells and skin," J. Invest. Dermatol. 127, 1498-1506 (2007).

54. M. K. Kuimova, S. W. Botchway, A. W. Parker, M. Balaz, H. A. Collins, H. L. Anderson, K. Suhling, P. R. Ogilby, "Imaging intracellular viscosity of a single cell during photoinduced cell death," Nat. Chem. 1, 69-73 (2009). 